\title{
The development of dynamic systems fault diagnosis technology
}

\section{Zhang Sheng $^{1}$, Cheng Zhengyong ${ }^{1}$, Li Ying ${ }^{1,2}$, He Banggui ${ }^{1,2}$, Gu Wenjuan ${ }^{1,2, a}$}

${ }^{1}$ Faculty of Mechanical and Electrical Engineering,Kunming University of Science and Technology, Kunming, Yunnan 650500, China

${ }^{2}$ Engineering Research Center of paper packaging and Printing in Yunnan Province, Kunming, Yunnan 650500, China

aEmail: guwenjuan001@163.com

Keywords: Dynamic fault diagnosis system, Fault diagnosis methods, Development Status

\begin{abstract}
After the rapid development of fault diagnosis technology for decades, there have been numerous methods based on different principles. For example, based on the analytical method of the model, the method based on signal processing and based on the method of knowledge. On the basis of comprehensive literature, this paper systematically summarizes the research status of the dynamic system of fault diagnosis technology at home and abroad.
\end{abstract}

\section{Introduction}

Dynamic system fault detection and diagnosis (FDD) is an application-oriented interdisciplinary. It involves modern control theory, computer engineering, signal processing, statistics, pattern recognition, artificial intelligence and corresponding application disciplines. Fault diagnosis is using the diagnosis system of all sorts of all sorts of state information and knowledge and integrated information processing. Finally we get on the comprehensive evaluation process of system operation and fault conditions.

\section{Fault diagnosis method based on the analytical model}

Analytical model based fault diagnosis method constructes residual signal using the system accurate mathematical model and the observed amount of input and output. Then we can find the desired behavior is inconsistent with the actual operation of the system between modes.

Based on the analytical model of fault diagnosis research is relatively more, also more deeply. Overall, these methods include tate estimation method, Parameter estimation method and Parity spacel method.

It has very good diagnostic effect using the deep understanding of internal system based on the analytical model of fault diagnosis. However, such method relies on the diagnostic object precise mathematical model. In practice, the object is often difficult to accurately establish the mathematical model. In this case the fault diagnosis method based on the analytical model is no longer applicable.

\section{The fault diagnosis method based on signal processing}

This kind of method is to extract fault related signal characteristics of time domain or frequency domain is used for fault diagnosis using the analysis and processing of various signal processing method for measuring signal, including spectral analysis method and wavelet transform method.

Different spectrum measurements will lead to failure of the signal exhibit different characteristics. So you can through to the signal power spectrum, cepstrum and spectrum analysis methods for fault diagnosis.

\section{Fault diagnosis method based on knowledge}

The late 80 s of the 20th century, with the rapid development of computer technology and 
artificial intelligence, fault diagnosis method based on knowledge was produced. And it has become the mainstream of the development direction of fault diagnosis and research.

Intelligent fault diagnosis system is mainly embodied in it can effectively acquisition, transmission, processing, regeneration, and make use of the diagnostic information. It has a capacity of a given environment, the correct diagnosis of the state of the object recognition, diagnosis and prediction. Intelligent Fault Diagnosis methods include:

(1) Diagnosis expert system-based approach.

Expert System is a System program with specialized knowledge and experience. It usually consists of the knowledge base, inference engine, human-machine interfaces and other parts. It is currently the most studied, the most widely used class of intelligent diagnostic techniques.

(2) Diagnostic method based on neural network.

Artificial Neural Network, ANN has massively parallel distributed processing, associative memory, self-organization learning characteristics, robustness and fault tolerance, etc. It has a very strong capacity for handling non-deterministic knowledge. It can solve many of the traditional methods can not solve the problem.

(3) Diagnostic method based on fuzzy theory.

Based on the theory of fuzzy diagnosis method does not need to establish accurate mathematical model, properly using membership function and fuzzy rules, fuzzy inference can implement the intelligent of the fuzzy diagnosis.

\section{The development status}

In recent years, with the development of signal processing and artificial intelligence disciplines, fault diagnosis technology has been evolving in the following aspects.

(1) Integrated development of intelligent fault diagnosis and fault diagnosis

Due to the complexity of the actual diagnosis of the problem, no single method can solve all diagnostic problems. Fusion to a variety of methods, giving full play to the advantages of various diagnosis methods, has become the inevitable trend of current fault diagnosis technology development. Such as the method of expert system, neural network and fuzzy combination, form the fuzzy neural network expert system. With the rapid development of artificial intelligence and pattern recognition theory, intelligent fault diagnosis has become a new research direction.

(2) The introduction of new methods such as genetic algorithms

Constantly enrich the content of the fault diagnosis technology, the genetic algorithm and artificial immune system such as the new method is successfully applied to the fault diagnosis and fault diagnosis technology will be the research focus. Genetic algorithm is a global optimization algorithm, a simple generic and robust, strong parallel processing capabilities, etc. Combination of genetic algorithms and neural networks is a noteworthy direction. In addition, the artificial immune system revealed by the natural immune system, is another powerful intelligent diagnostic method. Yang Jiangyun uses artificial immune system for rotating machinery fault diagnosis. And he completed the genetic algorithm and Troubleshooting.

(3) Knowledge representation and the development of automatic knowledge acquisition

Knowledge representation and automatic acquisition has been the difficulty intelligent fault diagnosis expert system research. In recent years, on the basis of the object-oriented programming, a technique called object-oriented knowledge representation developed. It provides a new way for solving the problem knowledge representation.

However, the way to solve the problem of automatic acquisition of knowledge is machine 
learning. Machine learning research is still in its primary stage at present, and is a need to develop research field of artificial intelligence. Appears with autonomy, self-adaptive intelligent body provides a good way to achieve this goal. In addition, the database system including information storage, sharing, concurrency control and recovery techniques to mature theoretical design knowledge base, improve diagnostic performance.

(4) Research of practical application and generalization of fault diagnosis software

A lot of research has been made in research on the theory of fault diagnosis results. But in the actual industrial process or device applications were rare. So the research engineering practical fault diagnosis system is worth attention. And now the existing fault diagnosis systems are mostly based on different devices and equipment development, respectively. Between each system architecture and development tools is differ in thousands ways. According to the actual need to develop a certain commonality of software, is also the focus of future research.

(5) The remote distributed fault diagnosis research

With the rapid development of automation technology, computer technology and network communication technology, The distributed remote diagnosis system, the realization of many experts on the same equipment in exotic collaborative diagnosis and multiple devices share the same diagnosis system, which can reduce the cost of the development of the system operation, improve the ability of fault diagnosis system of resources sharing. Research in this area will become an important element of fault diagnosis system research.

\section{Conclusion}

Fault diagnosis technology research is always new achievements in the field of learn from other disciplines on the basis of forward development. So we should follow the progress of each related disciplines and the latest research results applied to the fault diagnosis technology, opening up new ideas for the research of fault diagnosis.

\section{Acknowledgement}

In this paper, the research was sponsored by the Personnel Training Foundation of Yunnan Province (Project No. KKSY201201051).

\section{Reference}

[1] Zhou Donghua, Ye Yinzhong. Modern fault diagnosis and fault tolerant control. Beijing: Tsinghua University Press, 2000.

[2] Chen R H. Mingori D L. Speyer J L. Optimal Stochastic Fault Detection Filter[J]. Automatica, 2003,39:377-390.

[3] Yu-Dong Chen, Shi Songjiao, Weng Cheng Shin. Methods of dynamic system fault diagnosis [J]. Process Automation Instrumentation, 2001,28 (3) :1-14.

[4] xiao-ying liu, wei-hua GUI. The complex process of integrated intelligent fault diagnosis technology [J]. Journal of chemical industry automation and instrument, 2001, 28 (4) : 16.18.

[5] Wong Kai Ming, Qian Yu. Advances in chemical process fault diagnosis [J]. Process Automation Instrumentation, 2000,27 (3) :1-5. 